\title{
Propiedades psicométricas de la versión en español revisada del Inventario Alemán de Ansiedad ante Exámenes (GTAI- AR) en universitarios argentinos*
}

\section{Psychometric properties of the revised Spanish version of the German Test Anxiety Inventory (GTAI-AR) in argentinean university students}

Recibido: julio 8 de 2010 | Revisado: diciembre 14 de 2010 | Aceptado: enero 11 de 2011

\author{
Sebastián Eduardo Piemontesi ** \\ DANIEL ESTEBAN HEREDIA \\ LUIS AlBERTO FURLAN \\ Universidad Nacional de Córdoba, Argentina
}

SICI: 2011-2777(201203)11:1<177:GTAIAR>2.0.CO;2-O

Para citar este artículo: Piemontesi, S. E., Heredia, D. E. \& Furlan, L. A. (2012). Propiedades psicométricas de la versión en español revisada del Inventario Alemán de Ansiedad ante Exámenes (GTAI-AR) en universitarios argentinos. Universitas Psychologica, 11(1), 177-186.

* Artículo de invetigación.

Se agradece al Dr. Prof. Edgardo Pérez por su colaboración el la revisión de este artículo.

** Laboratorio de Evaluación Psicológica y Educativa. Enrique Barros esq. Enfermera Gordillo, Ciudad Universitaria, Córdoba (5000), Argentina. E-mails: piemontesi@psyche.unc.edu.ar,dheredia@psyche. unc.edu.ar, furlan@psyche.unc.edu.ar
RES UMEN

En el presente estudio se analizaron las propiedades psicométricas de la versión en español del Inventario Alemán de Ansiedad ante Exámenes (GTAI-AR) en universitarios argentinos. Se realizó un análisis factorial exploratorio y otro confirmatorio mediante parcelación de ítems. Se evaluó el ajuste de tres modelos de acuerdo a los antecedentes teóricos, uno que apoya la unidimensionalidad, otro que diferencia los componentes cognitivo y afectivo, y otro de cuatro dimensiones: Preocupación, Interferencia, Falta de Confianza y Emocionalidad como factores primer orden y la Ansiedad ante Exámenes total como factor de segundo orden. Los índices de ajuste para el último modelo fueron adecuados. Se discuten los resultados, el uso de parcelas de ítems y la importancia de contar con instrumentos multidimensionales de la Ansiedad ante Exámenes en lengua española.

Palabras clave autores:

Ansiedad ante exámenes, análisis factorial exploratorio, análisis factorial confirmatorio, parcelas de ítems.

Palabras clave descriptores:

Inventario Alemán de Ansiedad ante Exámenes (GTAI-AR), estudiantes universitarios, psicometría.

\footnotetext{
A B S T R A C T

In the present study was evaluated the psychometric properties of the Spanish version of the German Test Anxiety Inventory in argentinean university students. Was conducted an exploratory and a confirmatory factor analysis using items parcels. The fit of three possible models was evaluated according to the theoretical background, one that supports the unidimensionality of the test anxiety, other that distinguishes the cognitive and affective components, and one that comprising four dimensions, Worry, Interference, Lack of Confidence and Emotionality as primary factors and total Test Anxiety as secondary factor. Fit indices for the last model were all adequate. Are discussed the results, the use of items parcels and the importance of multidimensional instruments of test anxiety in Spanish language.

Key words authors:

Test Anxiety, exploratory factor analysis, confirmatory factor analysis, items parcels. Key words plus:

German Test Anxiety Inventory (GTAI-AR), university students, psychometry.
} 


\section{Introducción}

Las líneas más importantes de investigación sobre la Ansiedad ante Exámenes (AE) son la exploración de sus dimensiones, la evaluación psicométrica, las relaciones con el rendimiento académico u otras variables y la efectividad de los programas de tratamiento (Furlan \& Ponzo, 2006). Los desarrollos psicométricos resultan de gran importancia dado que contar con instrumentos adecuados permite evaluar con precisión a la AE así como conocer la efectividad de los programas de intervención en esta problemática, más aún teniendo en cuenta la prevalencia de la misma y los efectos que puede tener en la salud y el desempeño de los estudiantes (Heredia, Piemontesi, Furlan \& Hodapp, 2008).

La AE se define como una predisposición a experimentar elevada preocupación y activación fisiológica ante situaciones de evaluación. Los primeros hallazgos empíricos indicaron que consistía en una experiencia unitaria, donde elevados niveles están asociados a bajo rendimiento académico (Sarason \& Mandler, 1952). Posteriormente, se encontró que posee dos dimensiones, Preocupación y Emocionalidad y sólo la primera es la que afecta el rendimiento (Seipp, 1991; Zeidner, 1998). Esta distinción entre un componente cognitivo y un componente afectivo de la AE fue inicialmente introducida por Liebert y Morris (1967), sin embargo, diversos autores aportaron evidencias a favor del carácter multidimensional de la $\mathrm{AE}$ y, en este sentido, la clásica dimensión Preocupación fue reconocida como un constructo más heterogéneo y diferenciado (Rost \& Schermer, 1989; Schwarzer $\&$ Quast, 1985). Desde entonces, fueron realizados numerosos intentos para determinar cuáles y cuántas dimensiones necesitan ser distinguidas dentro del constructo general para comprender acabadamente el dominio de contenido de la AE.

En consecuencia, los instrumentos más recientes se componen de varias subescalas diseñadas para evaluar múltiples componentes de la AE (Morris, Davis \& Hutchings, 1981; Sarason, 1984). Siguiendo esta línea, el Inventario Alemán de Ansiedad ante Exámenes (German Test Anxiety Inventory [TAI-G], Hodapp, 1991) está conformado por las subescalas: Emocionalidad (referida a las percepciones de la activación fisiológica), Preocupación (referida a pensamientos sobre las consecuencias negativas de fracasar), Falta de Confianza (creencias negativas de la propia capacidad de rendir adecuadamente el examen) e Interferencia (pensamientos que producen distracción y bloqueo cognitivo), las cuales conforman un modelo de cuatro dimensiones que se agrupan en un factor de primer orden de AE. Tanto la escala total como las subescalas demostraron consistencia interna satisfactoria y diferentes estudios sobre la validez factorial, las relaciones de las cuatro subescalas con el rendimiento y otras variables han apoyado la conceptualización multidimensional de la AE evaluada mediante el TAI-G (Keith, Hodapp, SchermellehEngel \& Moosbrugger; 2003; Musch \& Broder, 1999). El GTAI-A, adaptación al español del TAI-G en estudiantes universitarios argentinos (Heredia et al., 2008), ha demostrado aceptable confiabilidad con coeficientes alfa de Cronbach entre 0.74 y 0.90 para cada una de las subescalas y la escala total, adecuada validez conservando la estructura factorial del instrumento original, correlaciones test-criterio con el rendimiento académico de - 0.34 con Interferencia y - 0.29 con Falta de Confianza, y diferencias de grupos contrastados con mayores puntajes en mujeres para Preocupación, Emocionalidad y Falta de Confianza. No obstante, para verificar la estructura interna de esta versión sólo se utilizó el análisis factorial exploratorio (AFE).

$\mathrm{El}$ análisis factorial confirmatorio (AFC) puede utilizarse para examinar la estructura factorial de las variables latentes y, en este sentido, es considerado un método eficiente para la validación transcultural de constructos psicológicos (Tomás Marco, González-Romá \& Gómez Benito, 2000). Usualmente, se utiliza el método de estimación de máxima verosimilitud [MV] (Hu \& Bentler, 1995; Yuan, Bentler \& Kano, 1997) para estimar los parámetros del modelo. El método de MV se basa en el supuesto de que los datos son continuos y están distribuidos normalmente, no obstante, algunos autores afirman que este supuesto es frecuentemente violado, especialmente cuando son analizados datos categóricos, (como las respuestas a ítems median- 
tes escalas tipo Likert, Flora \& Curran, 2004) y que además este método no provee una inferencia confiable cuando el número de variables analizado es excesivamente extenso (Nasser \& Takahashi, 2003). Por eso, tanto violar los supuestos del método MV como un excesivo número de variables, puede resultar en hallazgos engañosos o conclusiones inválidas sobre la estructura factorial (Bernstein \& Teng, 1989).

Una solución comúnmente empleada y que además ha demostrado ser útil para el estudio de la estructura factorial de la AE es la parcelación de ítems (Hagtvet \& Nasser, 2004; Nasser \& Takahashi, 2003; Nasser, Takahashi \& Benson, 1997; Prats, 1990). Las parcelas son utilizadas para reducir la complejidad y el número de parámetros a estimar del modelo, sumando o promediando grupos de ítems que se asume son conceptualmente similares y pertenecen a una única dimensión de un constructo (Thompson \& Melancon, 1996). Mediante este procedimiento, se construyen nuevas variables que resultan más continuas y que permiten una distribución más próxima de lo normal; por este motivo, la parcelación coincide con los supuestos del método MV (Bandalos \& Finney, 2001; Enders \& Bandalos, 1999). Se ha demostrado en estudios de AFC de inventarios de $\mathrm{AE}$ que la parcelación mejora las características de los nuevos indicadores (parcelas) y cuando el número de parcelas por factor es por lo menos tres, los resultados del AFC son consistentes (Nasser \& Takahashi, 2003; Nasser et al., 1997).

Considerando los argumentos anteriormente expuestos, el presente estudio tiene como objetivo evaluar la estructura de interna del GTAI-A en estudiantes universitarios, empleando el AFC mediante la parcelación de ítems. Si bien este trabajo aporta a la validación del inventario (fines instrumentales), posee una considerable utilidad práctica en el ámbito académico y clínico, ya que resulta de suma importancia conocer las manifestaciones que los estudiantes ansiosos experimentan para poder emprender programas de tratamiento eficientes que permitan mejorar su calidad de vida, tanto como la efectividad en el cursado de sus estudios.

\section{Método}

\section{Participantes}

En este estudio participaron 781 estudiantes universitarios con edades comprendidas entre los $17 \mathrm{y}$ 57 años $(M=22.5 ; D E=4.1)$. El $45.5 \%$ fueron hombres y el $52.2 \%$ mujeres, el $2.5 \%$ no lo informó. Los alumnos cursaban entre el primer y sexto año de sus carreras en las Facultades de Medicina (24.3\%), Economía (17.8 \%), Derecho (30.2\%) y Odontología (27.7 \%) en la Universidad Nacional de Córdoba, Argentina. Se trata de un muestreo no probabilístico, específicamente es un muestreo por conveniencia o accidental (Coolican, 1994) dado que la muestra se obtuvo de las Facultades donde sus autoridades accedieron formalmente a colaborar en la presente investigación.

\section{Instrumento}

Se utilizó el GTAI-A, adaptación al español del Inventario Alemán de Ansiedad frente a los Exámenes (Heredia et al., 2008), una medida de autoinforme de 28 ítems ( $\alpha=0.9$ para la escala total) comprendido por cuatro subescalas: Emocionalidad (8 ítems, $\mathbf{a}=0.88$ ), Preocupación (9 ítems, $\mathbf{a}=0.87$ ), Falta de Confianza (6 ítems de codificación inversa, $a=0.86$ ) e Interferencia ( 5 ítems, $\boldsymbol{a}=0.74$ ). Se realizaron algunas modificaciones a la versión adaptada original modificando la escala de respuesta tipo Likert de cuatro opciones por una de cinco opciones de respuesta que van de $1=$ nunca a $5=$ siempre, se incorporó un nuevo ítem a la escala de Interferencia y además fue revisada la redacción de los ítems número 4, 5, 15, 16, y 18 (véase Tabla 1).

\section{Procedimiento}

La administración de la escala fue colectiva y en momentos preestablecidos con la autorización de cada unidad académica; la participación fue voluntaria y se destacó el carácter confidencial de los resultados. Además, se brindó la posibilidad de recibir un reporte individual de los resultados 
a aquellos estudiantes interesados mediante un correo electrónico.

\section{Análisis de datos}

Dadas las modificaciones realizadas, se verificó en primer lugar la estructura interna del GTAI-A mediante el AFE (Ejes principales, rotación Promax) con el programa SPSS 11.

En segundo lugar, las parcelas se crearon combinando métodos teóricos y empíricos. La unidimensionalidad de las escalas es el prerrequisito más importante para la creación de parcelas (Matsunaga, 2008), por esto se exploró la pertenencia de los ítems a una misma dimensión de la AE basándonos en: 1) el modelo teórico de cuatro dimensiones de Hodapp (1991), 2) el contenido del ítem y su redacción y 3) el AFE. Además, para optimizar la normalidad de las parcelas se evaluaron 4) los índices de simetría de los ítems. Sobre la base de lo anterior, se utilizó el método de parcelación aislada (isolated parceling, Holt, 2004) entre los ítems de cada dimensión y fueron agrupados aquellos con índices de simetría más negativos con los más positivos de acuerdo al procedimiento usado por Thompson y Melancon (1996) y sugerido por Holt (2004).

En tercer lugar, el AFC fue realizado utilizando el método de estimación de MV mediante el programa AMOS 4 (Arbuckle \& Wothke, 1999). Para evaluar el ajuste de los modelos comparados se utilizaron múltiples indicadores ( $\mathrm{Hu} \&$ Bentler, 1995). Específicamente, se utilizó el estadístico chi cuadrado, la razón de chi cuadrado sobre los grados de libertad (CMIN/DF), el cambio en chi cuadrado de los modelos alternativos, el índice de tras-validación supuesta (ECVI), el índice de ajuste comparativo (CFI), el índice de ajuste no normativo o Tucker-Lewis (TLI), el índice de bondad del ajuste global (GFI) y el error cuadrado de aproximación a las raíces medias (RMSEA).

Utilizando el procedimiento del programa SPSS para generar muestras aleatorias, se dividió la muestra en dos mitades que incluyeron el $50 \%$ de los participantes. Se verificaron los datos incompletos, los casos atípicos univariados y multivariados y el grado de curtosis y simetría de las variables (Tabachnick \& Fidell, 2001). Luego de dividir la muestra, se aplicó el AFE en una mitad y la segunda mitad de la muestra fue utilizada para realizar el AFC. La consistencia interna de las escalas del instrumento se estimó mediante el coeficiente alfa de Cronbach.

\section{Resultados}

Los valores de la medida de adecuación muestral Kaiser-Meyer-Olkin $(=0.915)$ y la prueba de esfericidad de Bartlett (4904.05; $d f=378 ; p=0.000$ ) indicaron la posibilidad de realizar el AFE, el cual se efectuó a través del método de extracción de Ejes Principales. Tanto la regla de Kaiser-Guttman, la interpretación del Scree Test (Cattell, 1966) y el Análisis Paralelo (Horn, 1965) indicaron la conveniencia de interpretar cuatro factores.

Por consiguiente se extrajeron 4 factores que explicaron un 55 \% de la varianza. Se realizó una rotación oblicua Promax (Tabachnick \& Fidell, 2001) dada la correlación entre las dimensiones de la $\mathrm{AE}$ (mayores a 0.3). Como criterios complementarios se decidieron retener aquellos ítems con una saturación factorial superior a 0.3 . Si un ítem presenta correlaciones por encima de 0.3 en dos factores, se considerará que contribuye al factor en que la saturación es mayor, siempre que la diferencia entre las cargas factoriales del ítem en cuestión sea mayor que 0.1. Como resultado se obtuvo una solución de 29 ítems distribuidos en 4 factores con autovalores superiores a 1 que explicaron el $55.5 \%$ de la varianza de respuesta al test. Esto fue confirmado tanto por el gráfico Scree y como el Análisis Paralelo. Los factores extraídos fueron rotados utilizando el método Promax. Del estudio de la matriz de correlaciones ítem-factor pudo observarse que el ítem 22 poseía una carga mayor a 0.3 en dos factores, sin embargo la diferencia de correlación fue mayor a 0.1 por lo cual fue retenido. El inventario quedó finalmente conformado por 4 subescalas Emocionalidad ( $a=0.87)$, Preocupación $(a=0.82)$, Falta de Confianza $(a=89)$; Interferencia $(a=0.87)$ y la escala de AE Total $(a=0.91)$ conformada por la sumatoria de las cuatro anteriores (véase la Tabla 1). 
TABLA 1

Matriz de correlaciones ítem-factor

\begin{tabular}{|c|c|c|c|c|c|}
\hline № & Ítem. & Factor-1 & Factor-2 & Factor-3 & Factor-4 \\
\hline 7 & Tengo confianza en mi propio desempeño & 0.877 & -0.001 & -0.016 & -0.033 \\
\hline 28 & Estoy convencido de que haré bien el examen & 0.829 & -0.028 & -0.013 & -0.065 \\
\hline 12 & Sé que puedo confiar en mí mismo & 0.808 & -0.02 & 0.048 & -0.048 \\
\hline 1 & Tengo seguridad en mi capacidad & 0.786 & 0.072 & -0.049 & -0.006 \\
\hline 25 & Confío que lograré hacerlo todo & 0.728 & -0.065 & -0.099 & -0.018 \\
\hline 19 & Me siento conforme conmigo mismo & 0.705 & 0.006 & 0.001 & -0.005 \\
\hline 3 & Tengo una sensación rara en mi estómago & -0.041 & 0.881 & -0.046 & -0.245 \\
\hline 27 & Me siento nervioso & 0.013 & 0.796 & 0.049 & -0.066 \\
\hline 15 & Siento que mi corazón late fuerte & -0.034 & 0.781 & -0.116 & 0.08 \\
\hline 6 & Siento mi cuerpo tensionado & -0.044 & 0.771 & -0.037 & 0.059 \\
\hline 21 & Tiemblo de nerviosismo & -0.026 & 0.569 & 0.002 & 0.163 \\
\hline 11 & Me siento intranquilo & 0.084 & 0.483 & -0.002 & 0.121 \\
\hline 17 & Me siento ansioso & -0.042 & 0.481 & 0.262 & -0.088 \\
\hline 24 & Tengo una sensación de angustia & 0.134 & 0.467 & 0.008 & 0.234 \\
\hline 13 & Pienso en lo mucho que me importa obtener un buen resultado & -0.127 & -0.035 & 0.732 & -0.09 \\
\hline 16 & Me preocupa el resultado de mi examen & 0.027 & 0.002 & 0.682 & -0.083 \\
\hline 20 & Me preocupa cómo se verá mi calificación & -0.006 & -0.034 & 0.654 & -0.07 \\
\hline 26 & Pienso en lo que pasará si me va mal & 0.076 & -0.039 & 0.616 & 0.065 \\
\hline 9 & Me pregunto si mi rendimiento será lo suficientemente bueno & -0.003 & -0.023 & 0.57 & 0.093 \\
\hline 8 & Pienso en las consecuencias de fracasar & 0.108 & -0.106 & 0.566 & 0.212 \\
\hline 22 & Me preocupa que algo pueda salirme mal & 0.043 & 0.346 & 0.49 & -0.057 \\
\hline 2 & Pienso en la importancia que tiene el examen para mí & -0.246 & 0.018 & 0.47 & -0.061 \\
\hline 5 & Me preocupa saber si podré hacer bien el examen & 0.123 & 0.172 & 0.33 & 0.072 \\
\hline 29 & $\begin{array}{l}\text { Me invade cualquier pensamiento haciendo difícil que me concen- } \\
\text { tre }\end{array}$ & 0.101 & -0.046 & 0.078 & 0.802 \\
\hline 14 & Fácilmente pierdo el hilo de mis pensamientos & 0.071 & -0.097 & -0.018 & 0.759 \\
\hline 23 & $\begin{array}{l}\text { Me llama la atención algo de poca importancia que interrumpe mi } \\
\text { razonamiento }\end{array}$ & -0.071 & -0.041 & 0.052 & 0.708 \\
\hline 10 & Pienso en cualquier cosa y me distraigo & -0.152 & -0.035 & -0.089 & 0.681 \\
\hline 18 & Otros problemas me interfieren y no puedo recordar las cosas & -0.007 & 0.069 & -0.055 & 0.64 \\
\hline 4 & Se me cruzan pensamientos por la cabeza que me bloquean & 0.043 & 0.246 & 0.099 & 0.398 \\
\hline a & Escala Total: 0.91 & 0.89 & 0.87 & 0.82 & 0.87 \\
\hline
\end{tabular}

Nota. $N=389$

Fuente elaboración propia.

Obtenidos los resultados del AFE se procedió a la creación de parcelas de ítems. Utilizando los criterios anteriormente expuestos las parcelas quedaron conformadas de la siguiente manera: (A) Preocupación: P.1 = ítems 16, 20 у 22; P.2 = ítems 2, 5 y 13; P.3 = ítems 8,9 y 26. (B) Falta de confianza: FC.1 = ítems 7 y 28 , FC. $2=$ items 1 y 25 , FC.3 = ítems 12 y 19. (C) Emocionalidad: E.1 = ítems 6, 17 y 21; E.2 = ítems
3, 24 y 27; E.3 = ítems 11 y 15. (D) Interferencia: $\mathrm{I} .1=$ ítems 4 y 10; I.2 = ítems 14 y 23; I.3 = ítem 18 y 29. Seguidamente, se exploraron los índices de asimetría y curtosis para cada parcela, obteniendo resultados adecuados para todas en ambos índices; además se exploró la consistencia interna de cada grupo de parcelas obteniéndose también valores adecuados, en especial considerando el reducido número de parcelas incluidas (3 parcelas, véase Tabla 2). 
TABLA 2

Estadística descriptiva de las parcelas

\begin{tabular}{lccccc}
\hline \multicolumn{1}{c}{ Parcela } & $\mathrm{M}$ & $\mathrm{DE}$ & Simetría & Curtosis & Alfa \\
\hline P1 & 11.73 & 2.47 & -0.46 & -0.56 & \\
P2 & 12.04 & 2.02 & -0.36 & -0.45 & $\mathrm{a}=0.78$ \\
P3 & 10.84 & 2.94 & -0.33 & -0.78 & \\
\hline FC1 & 4.89 & 1.76 & 0.42 & 0.18 & \\
FC2 & 4.85 & 1.71 & 0.34 & -0.16 & $\mathrm{a}=0.9$ \\
FC3 & 4.78 & 1.76 & 0.38 & -0.13 & \\
\hline E1 & 10.26 & 2.77 & -0.03 & -0.70 & \\
E2 & 10.23 & 3.09 & -0.26 & -0.68 & $\mathrm{a}=0.88$ \\
E3 & 6.61 & 2.15 & -0.03 & -0.90 & \\
\hline I1 & 5.46 & 1.77 & 0.27 & -0.07 & \\
I2 & 4.80 & 1.94 & 0.64 & -0.12 & $\mathrm{a}=0.8$ \\
I3 & 4.41 & 1.75 & 0.73 & -0.28 & \\
\hline
\end{tabular}

Nota. $N=392$

Fuente elaboración propia.

Por último, se realizó el AFC para verificar el ajuste a de los datos a 4 modelos diferentes. El primer modelo pone a prueba la unidimensionalidad de la AE y se basa en la concepción clásica (Sarason \& Mandler, 1952; véase Figura 1). El segundo que diferencia el componente cognitivo y afectivo que están correlacionados, está basado en la concepción bidimensional (Liebert \& Morris, 1967), por ello se agruparon Preocupación, Interferencia y Falta de Confianza para formar el componente cognitivo y Emocionalidad conformó el componente afectivo (Figura 2). El tercer modelo coincide con el propuesto por el autor del instrumento (Hodapp, 1991), donde las variables observadas son explicadas por cuatro variables latentes de primer orden que representan cada dimensión y que a su vez son explicadas por una única variable latente de segundo orden de AE (Figura 3). Adicionalmente, se analizaron los datos de ajuste de un cuarto modelo alternativo de independencia o nulo.

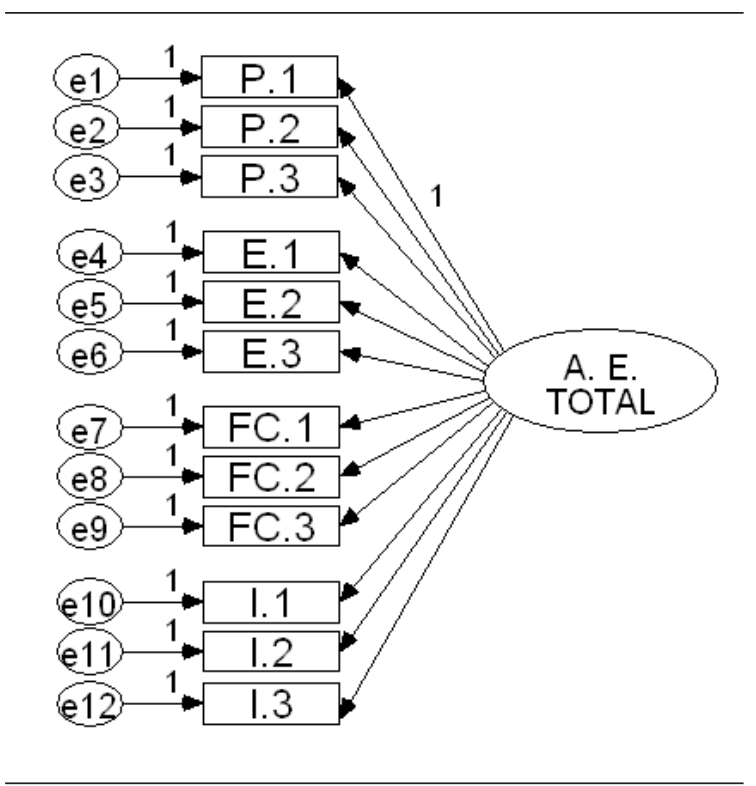

Figura 1. Modelo alternativo 1.

A. E. Total $=$ ansiedad ante los exámenes

Fuente: elaboración propia.

Se exploró la razón de chi cuadrado sobre los grados de libertad, con valores inferiores a 3 indicando un buen ajuste. El cambio significativo en chi cuadrado indica que un modelo ajusta mejor que el 


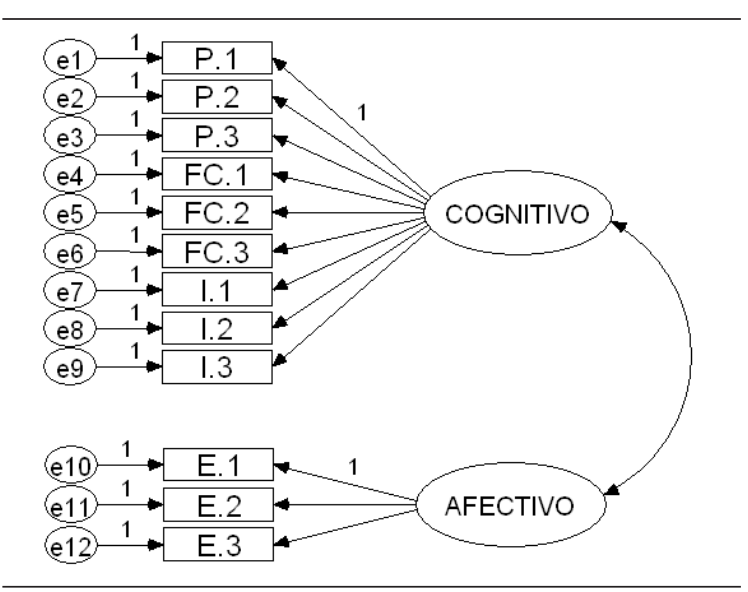

Figura 2. Modelo alternativo 2.

Fuente: elaboración propia.

anterior (Jöreskog \& Sörbom, 1993) y los puntajes del ECVI permiten elegir entre modelos; los valores menores indican el modelo con mejor ajuste a los datos y más parsimonioso (Weston \& Gore 2006). Para los índices CFI, GFI y TLI los valores 0.95 o superiores son considerados excelentes, y valores superiores a 0.9 sugieren un ajuste aceptable. El índice RMSEA es considerado óptimo cuando sus valores son de 0.05 o inferiores y aceptables en el rango

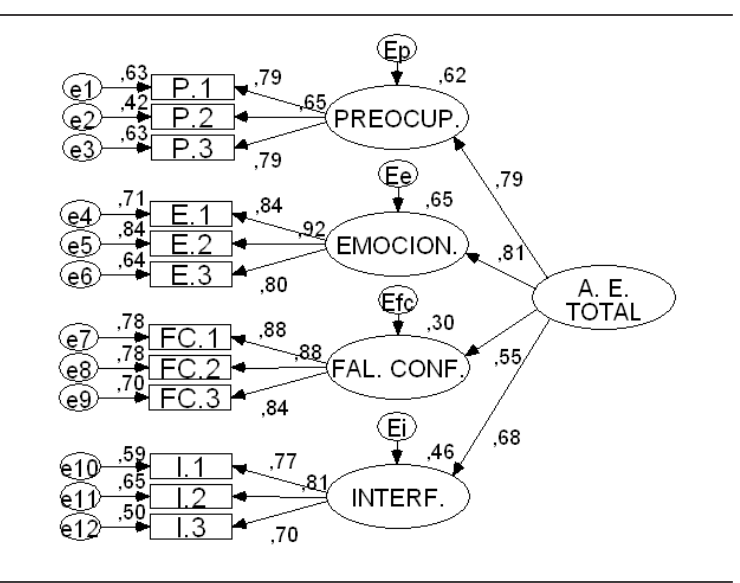

Figura 3. Análisis factorial confirmatorio del GTAI-AR. Modelo $3(N=392)$. A. E. Total = ansiedad ante los exámenes

Fuente: elaboración propia.

0.08-0.05 (Hu \& Bentler, 1995). Comparando los índices de ajuste de los diferentes modelos, puede apreciarse (Tabla 3) que el modelo de cuatro variables latentes de primer orden y una variable latente de segundo orden ajusta aceptablemente (GFI 0.94; TLI 0.95; CFI 0.97; ECVI 0.50; RMSEA 0.067), y de manera significativamente superior (cambio significativo en chi cuadrado) a los restantes modelos.

\section{TABLA 3}

Índices de ajuste de los cuatro modelos del TAI-G

\begin{tabular}{lccccccccc}
\hline & \multicolumn{10}{c}{ Índices de Ajuste } \\
\cline { 2 - 9 } Modelos & $X^{2}$ & $d f$ & CMIN/DF & GFI & TLI & CFI & ECVI & RMSEA & Dif. $X^{2}$ \\
\hline Nulo & $2631.564 * * *$ & 66 & 39.872 & 0.35 & - & - & 6.79 & 0.315 & \\
Modelo 1 & $1134.349 * * *$ & 54 & 21.006 & 0.64 & 0.48 & 0.58 & 3.02 & 0.226 & $1497.215^{* * *}$ \\
Modelo 2 & $825.246^{* * *}$ & 53 & 15.571 & 0.67 & 0.62 & 0.70 & 2.01 & 0.193 & $309.103 * * *$ \\
Modelo 3 & $138.559^{* * *}$ & 50 & 2.771 & 0.94 & 0.95 & 0.97 & 0.5 & 0.067 & $686.687 * * *$ \\
\hline
\end{tabular}

Nota $X^{2}=$ chi cuadrado; $d f=$ grados de libertad; CMIN/DF = chi cuadrado sobre grados de libertad; GFI = Índice de bondad del ajuste; TLI = índice de ajuste no normalizado; CFI = índice de ajuste comparativo; ECVI = índice de tras-validación supuesta; RMSEA = Error cuadrado de aproximación a las raíces medias; $N=392 ; * * * p<0.001$

Fuente: elaboración propia. 


\section{Discusión}

El GTAI-AR ha demostrado validez y fiabilidad para evaluar las múltiples dimensiones de la AE. La importancia de explorar las distintas manifestaciones que los estudiantes experimentan radica en que permitiría adecuar programas de tratamiento más eficientes. Este hecho es relevante dado que las diferencias individuales en la manifestación de cada dimensión están relacionadas de manera específica a distintas estrategias de estudio y de afrontamiento y a diferentes niveles de autoeficacia para la autorregulación del aprendizaje y de rendimiento académico (Furlan, Sánchez Rosas, Heredia, Piemontesi \& Illbele, 2009; Piemontesi \& Heredia, 2009).

Investigaciones recientes han demostrado que, si bien los estudiantes con elevada AE presentan una tendencia a usar estrategias de estudio más superficiales de procesamiento y los de baja a utilizar estrategias más reflexivas y críticas (Furlan et al., 2009; Naveh-Bemjamin, McKeachie \& Lin, 1987; Zeidner, 1998), cuando se consideran las distintas dimensiones puede observarse que la Preocupación está asociada al uso de estrategias orientadas a aprovechar el tiempo, administrar el esfuerzo y fijar conocimientos, por lo que podría afirmarse que la misma tiende a movilizar recursos que mejoran la preparación (Eysenck \& Gutiérrez Calvo, 1992). Esta tendencia también se observó en otros trabajos donde la Preocupación se relacionó con el uso de estrategias de afrontamiento orientadas al problema y mayor autoeficacia para la autorregulación de aprendizaje (Piemontesi \& Heredia, 2009; Stöber, 2004). Al contrario, el aumento de la Falta de Confianza se asocia a la disminución en el uso general de estrategias de estudio y promueve de algún modo conductas de evitación y procrastinación. Por otra parte, cuando hay elevada Interferencia aparecen dificultades para aprovechar el tiempo, sostener el esfuerzo, y aumenta el uso de estrategias superficiales como la repetición (Furlan et al., 2009). Estos datos ponen de manifiesto que para las intervenciones terapéuticas de la $\mathrm{AE}$ se debe contar con recursos que permitan atender a cada una de estas manifestaciones.
El objetivo de este estudio fue validar mediante AFC y el uso de parcelas de ítems la versión revisada de la adaptación argentina del Inventario Alemán de Ansiedad ante Exámenes. El GTAI-AR fue administrado a una muestra de estudiantes universitarios de diferentes carreras y se analizó la estructura interna y la confiabilidad. El AFE brindó soporte empírico para la estructura de cuatro dimensiones de la AE propuesta por el autor del instrumento (Hodapp, 1991) con consistencias internas adecuadas. La creación de las parcelas fue realizada siguiendo tanto criterios teóricos como empíricos para asegurar que los ítems agrupados pertenezcan a una misma dimensión. Los resultados del AFC demostraron que el modelo de cuatro variables latentes de primer orden y una de segundo orden es el que se ajusta de manera aceptable a los datos y es congruente con el modelo de Hodapp de la AE.

Entre las limitaciones pueden considerarse las posibles dificultades del uso de parcelas las cuales pueden ser menos informativas que el uso de ítems individuales como indicadores, pero este problema solamente ha sido informado para el AFC de evaluación de equivalencias o de invarianza de medición, una metodología utilizada cuando se mide un atributo bajo distintas condiciones como la estabilidad en el tiempo, a través de diferentes poblaciones, grupos o modos de administración (Meade \& Kroustalis, 2005), para el cual la parcelación no es recomendada.

Se plantea para estudios futuros verificar la estabilidad temporal del instrumento. Si bien existen investigaciones que destacan la concepción de la $\mathrm{AE}$ evaluada mediante el TAI-G como un rasgo, lo que implicaría que su manifestación depende más de la persona que de la situación, sería de suma utilidad poner a prueba esta concepción con la adaptación argentina del instrumento mediante la teoría del "Rasgo-estado latente" (Keith et al., 2003) la cual brinda medios más sofisticados para evaluar rasgos que los métodos convencionales tales como el testretest. Además, se propone para próximos estudios evaluar la invarianza transcultural del constructo en muestras alemanas y argentinas.

No obstante, puede concluirse que el GTAIAR posee propiedades psicométricas adecuadas 
de consistencia interna y estructura factorial. En suma, los presentes resultados suministran apoyo al GTAI-AR como un instrumento potencialmente útil para explorar las distintas manifestaciones de la Ansiedad ante Exámenes de los estudiantes universitarios argentinos, tanto como para evaluar de manera fiable los programas de tratamiento e intervención de esta dificultad, en lengua española.

\section{Referencias}

Arbuckle, J. \& Wothke, W. (1999). AMOS users guide version 4.0. Chicago: SmallWaters.

Bandalos, D. \& Finney, J. S. (2001). Item parceling issues in structural equation modeling. En A. G. Marcoulides \& E. R. Schumacker (Eds.), New developments and techniques in structural equation modeling (pp.269-295). Hillsdale, NJ: Lawrence Erlbaum Associates, Inc.

Bernstein, H. \& Teng, G. (1989). Factoring items and factoring scales are different: Spurious evidence for multidimensionality due to item categorization. Psychological Bulletin, 105, 467-477.

Cattell, R. (1966). The Scree Test for the number of factors. Multivariate Behavioral Research, 1, 141-161.

Coolican, H. (1994). Métodos de investigación y estadística en psicología. México: El Manual Moderno.

Enders, K. C. \& Bandalos, L. D. (1999). The effects of heterogeneous item distribution on reliability. Applied Measurement in Education, 12, 133-150.

Eysenck, M. W. \& Gutiérrez Calvo, M. (1992). Anxiety and performance: The processing efficiency theory. Cognition and Emotion, 6, 409-434.

Flora, D. B. \& Curran, P. J. (2004). An empirical evaluation of alternative methods of estimation for confirmatory factor analysis with ordinal data. Psychological Methods, 9(4), 466-491

Furlan, L. \& Ponzo, A. (2006). Relaciones entre ansiedad de evaluación y rendimiento académico. En Memorias de las XIII Jornadas de Investigación y Segundo Encuentro de Investigadores en Psicología del MERCOSUR (pp. 236-238). Buenos Aires: Facultad de Psicología, Universidad de Buenos Aires.

Furlan, L., Sánchez-Rosas, J., Heredia, D., Piemontesi, S. \& Illbele, A (2009). Estrategias de aprendizaje y ansiedad ante los exámenes en estudiantes universitarios. Pensamiento Psicológico, 12, 117-124.

Hagtvet, K. A. \& Nasser, F. M. (2004). How well do item parcels represent conceptually defined latent constructs? A two-facet approach. Structural Equation Modeling: A Multidisciplinary Journal, 11, 168-193.

Heredia, D., Piemontesi, S., Furlan, L. \& Hodapp, V. (2008). GTAI-A: adaptación del Inventario Alemán de Ansiedad Frente a los Exámenes. Evaluar, 8, 46-60.

Hodapp, V. (1991). Das Prüfungsängstlichkeitsinventar TAI-G: Eine erweiterte und modifizierte Version mit vier Komponenten [The Test Anxiety Inventory TAI-G: An expanded and modified version with four components]. Zeitschrift für Pädagogische Psychologie, 5, 121-130.

Holt, J. K. (2004, octubre). Item parceling in structural equation models for optimum solutions. Paper presented at the 2004 Annual Meeting of the Mid-Western Educational Research Association, Columbus, OH, United States.

Horn, J.L. (1965). A rationale and test for the number of factors in factor analysis. Psychometrika, 30, 179-185.

Hu, L. \& Bentler, P. M. (1995). Evaluating model fit. En R. Hoyle (Ed.), Structural equation modeling: Concepts, issues and applications (pp.76-99). Thousand Oaks, CA: Sage Publications.

Hu, L. \& Bentler, P. M. (1999). Cutoff criteria for fit indexes in covariance structure analysis: Conventional criteria versus new alternatives. Structural Equation Modeling, 6(1), 1-55.

Jöreskog, K. \& Sörbom, D. (1993). LISREL 8: User's reference guide. Chicago, IL: Scientific Software International.

Keith, N., Hodapp, V., Schermelleh-Engel, K. \& Moosbrugger, H. (2003). Crossectional and longitudinal confirmatory factor models for the German Test Anxiety Inventory: A construct validation. Anxiety, Stress, and Coping, 16, 251-270.

Liebert, R. M. \& Morris, L. W. (1967). Cognitive and emotional components of test anxiety: A distinction and some initial data. Psychological Reports, 20, 975-978. 
Matsunaga, M. (2008). Item parceling in structural equation modeling: A Primer. Communication Methods and Measures, 2(4), 260-293.

Meade, A. W. \& Kroustalis, C. M. (2005, abril). Problems with item parceling for confirmatory factor analysis tests of measurement invariance of factor loadings. Paper presented at the 20th Annual Conference of the Society for Industrial and Organizational Psychology, Los Angeles, CA, United States.

Morris, L. W., Davis, M. A. \& Hutchings, C. H. (1981). Cognitive and emotional components of anxiety: Literature review and a revised worry-emotionality scale. Journal of Educational Psychology, 73, 541-555.

Musch, J. \& Broder, A. (1999). Uberprufung der psychometrischen Eigenschaften und der Validitat des multidimensionalen Prufungsangstlichkeitsinventars TAI-G [Investigating the psychometric properties and the validity of the multidimensional German Test Anxiety Inventory TAI-G]. Zeitschrift fur Padagogische Psychologie, 13, 100-105.

Nasser, F. \& Takahashi, T. (2003). The effect of using item parcels on ad hoc goodness-of-fit indexes in confirmatory factor analysis: An Example using Sarason's reactions to tests. Applied Measurement in Education, 16(1), 75-97.

Nasser, F., Takahashi, T. \& Benson, J. (1997). The structure of test anxiety in Israeli-Arab high school students: An application of confirmatory analysis with miniscales. Anxiety, Stress, and Coping, 10, 129-151.

Naveh-Benjamin, M., McKeachie, W. \& Lin, Y. (1987). Two types of test-anxious students: Support for an information processing model. Journal of Educational Psychology, 79, 131-136.

Piemontesi, S. E. \& Heredia, D. E. (2009). Correlatos de la ansiedad ante los exámenes: una aproximación a la teoría de la reducción en la eficiencia. Revista Tesis, 2, 74-86.

Prats, D. C. (1990, abril). The effects of forming miniscales on the construct validity of the Test Anxiety Inventory. Paper presented at the annual meetings of the National Council of Measurement of Education, Boston, MA., Unites States.

Rost, D. H. \& Schermer, F. J. (1989). The various facets of test anxiety: A subcomponent model of test anxiety measurement. En R. Schwartzer, H. M. Van der Ploeg \& C. D. Spielberger (Eds.), Advances in test anxiety research (Vol. 6, pp. 37-52 ). Lisse: Swets \& Zeitlinger.

Sarason, I. G. (1984). Stress, anxiety, and cognitive interference: Reactions to tests. Journal of Personality and Social Psychology, 46, 929-938.

Sarason, S. B. \& Mandler, G. (1952). Some correlates of test anxiety. Journal of Consulting and Clinical Psychology, 47, 810-817.

Schwarzer, R. \& Quast, H. H. (1985). Multidimensionalty of the anxiety experience: Evidence for additional components. En H. M. van der Ploeg, R. Schwarzer \& C. D. Spielberger (Eds.), Advances in Test Anxiety Research (pp.3-14). Lisse: Swets \& Zeitlinger.

Seipp, B. (1991). Anxiety and academic performance: A meta-analysis of findings. Anxiety Research, 4, $27-41$.

Stöber, J. (2004). Dimensions of test anxiety: Relations to ways of coping with pre-exam anxiety and uncertainty. Anxiety, Stress, and Coping, 17(3), 213-226.

Tabachnick, B. \& Fidell, L. (2001). Using multivariate statistics (4a. ed.). Boston: Allyn and Bacon.

Thompson, B. \& Melancon, J. (1996, noviembre). Using item "testlets/parcels" in confirmatory factor analysis: An example using the PPDP-78. Paper presented at the annual meeting of the Mid-South Educational Research Association, Tuscaloosa, AL, United States.

Tomás Marco, I., González-Romá, V. \& Gómez Benito, J. (2000). Teoría de respuesta al ítem y análisis factorial confirmatorio: dos métodos para analizar la equivalencia psicométrica en la traducción de cuestionarios. Psicothema, 12(2), 540-544.

Weston, R. \& Gore, P. A. (2006). A brief guide to structural equation modeling. The Counseling Psychologist, 34(5), 719-751.

Yuan, K. H., Bentler, P. M. \& Kano, Y. (1997). On averaging variables in a confirmatory factor análisis model. Behaviormetrika, 24, 71-83.

Zeidner, M. (1998). Test anxiety: The state of the art. New York/London: Plenum Press. 